\section{Oral Programme}

Parallel Session A

Wednesday 12 September 2012

\section{Socioeconomic Inequalities I}

\section{OP01 DID HEALTH INEQUALITY INCREASE IN ENGLISH CHILDREN AND YOUNG PEOPLE BETWEEN 1999 AND 2009? EVIDENCE FROM TWO CROSS-SECTIONAL SURVEYS AND INPATIENT ACTIVITY DATA}

doi:10.1136/jech-2012-201753.001

${ }^{1}$ DS Hargreaves, ${ }^{2}$ A Djafari-Marbini. ${ }^{1}$ General and Adolescent Paediatrics, University College London (UCL) Institute of Child Health, London, UK; '2Speciality Services Directorate, Imperial College Healthcare NHS Trust, London, UK

Background From 1999, the English government pursued a systematic strategy to reduce health inequalities. For interventions affecting children and young people, intermediate indicators may be more useful for evaluating short/medium term impact than the mortality targets chosen. This article investigates trends in inequalities for self/parent-reported health and use of health services by children and young people between 1999 and 2009.

Methods Through the UK Data Archive (http://www.dataarchive.ac.uk/), data were accessed for the Health Survey for England 1999 (SN4365) ( $\mathrm{N}=2638(0-12$ years), 874(13-16), 1148(17-24), 5573(25+)) and the Health Survey for England 2009 (SN6732) (N=3022(0-12), 969(13-16), 451(17-24), 4160(25+)). Self-reported health and General Health Questionnaire data (participants aged 13+) and parent/carer report of health (participants aged 0-12) were used, with appropriate binary outcomes created. Using logistic regression in SPSS (v19), adjusted odds ratios (AORs) of poor health were calculated between the highest and lowest socio-economic tertiles, defined by occupation of household members. Analyses were stratified by age, adjusted for sex and weighted to be nationally representative. Hospital Episode Statistics on Finished Consultant Episodes were analysed by Index of Multiple Deprivation decile, derived from the patient's postcode. Concentration indices of inpatient activity were calculated for those aged $0-12$ and $13-19$ in 1999/2000 ( $\mathrm{N}=1843862)$ and 2009/2010 ( $\mathrm{N}=1774139)$.

Results Compared to those in the highest occupations, parents/ carers in the lowest tertile were more likely to report poor health in their children. The difference increased significantly from 1999 (AOR 1.7, 95\% CI 1.2-2.4) to 2009 (AOR 4.1, 95\% CI 2.5 to 6.7). Among adolescents, there was a non-significant increase in poor self-reported health over this time (AOR 2.3(1.2-4.3) vs. 3.2(1.6$6.4)$ ). For young adults, there was a persistently weaker association between low socio-economic status and poor health (AOR 1.3(0.8$2.2)$ and 1.3(0.7-2.4)) than for older adults (AOR 3.1(2.6-3.6) and 3.2(2.6-3.9)). AORs for high General Health Questionnaire scores by age group showed a similar pattern.

The concentration index for inpatient activity in adolescents (13-19) decreased from -0.102 in 1999 to -0.082 in 2009 (Males) and -0.247 to -0.189 (Females). The changes in the concentration index for $0-12$ s were much smaller (Male -0.136 vs. -0.139 ; Female -0.136 vs. -0.142 ).

Conclusion Despite the policy importance given to tackling health inequalities, this decade saw inequality in parents' reports of their children's health widen significantly. Adolescent findings were mixed, with a non-significant increase in inequality for selfreported health but a reduction in inequality related to inpatient activity.
SOCIOECONOMIC PATTERNING OF FAT AND LEAN MASS IN LATER LIFE: FINDINGS FROM A BRITISH BIRTH COHORT STUDY

doi:10.1136/jech-2012-201753.002

'D Bann, 'R Cooper, ${ }^{2} \mathrm{~A}$ Wills, ${ }^{3} \mathrm{~J}$ Adams, ${ }^{1} \mathrm{D}$ Kuh. ${ }^{1} \mathrm{MRC}$ Unit for Lifelong Health and Ageing, Division of Population Health, UCL, London, UK; ${ }^{2}$ MRC CAiTE, School of Social \& Community Medicine, University of Bristol, Bristol, UK; ${ }^{3}$ Clinical Radiology, Manchester Royal Infirmary, Oxford Road, Manchester, UK

Background Fat and lean mass have important implications for health and physical functioning. Studies have reported associations between low socioeconomic position (SEP) and high body mass index (BMI) in adulthood, but few have examined associations with fat and lean mass which BMI does not completely distinguish. We examined the associations between multiple indicators of SEP across life and objective measures of fat and lean mass in later adulthood.

Methods Data were from 1350 men and women in England, Scotland and Wales participating in the MRC National Survey of Health and Development who underwent dual-energy X-ray absorptiometry scans at age 60-64 years and had valid data for all indicators of SEP were included in analyses. Whole body fat and appendicular lean mass were ascertained and, to account for differences in height across SEP groups, height-adjusted indices were created. Associations of prospectively ascertained childhood SEP (paternal occupational class at 4 years), educational attainment (at 26 years) and adult SEP (household income at 60-64 years) with these outcomes were examined using the slope index of inequality (SII). Since high fat mass may increase muscle loading and lead to higher lean mass, associations with lean mass were adjusted for fat mass.

Results Low SEP was consistently associated with high fat mass index: childhood SEP (mean difference in fat mass index comparing the lowest with the highest SEP (SII) in males: $0.92 \mathrm{~kg} / \mathrm{m}^{1.2}, 95 \%$ CI -0.04 to 1.89 ; females: $2.49,1.17$ to 3.82 ), education (males: 1.61 , 0.69 to 2.53 ; females: $2.20,0.88$ to 3.53 ), and adult SEP (males: 1.42 , 0.47 to 2.38; females: $2.20,0.88$ to 3.53 ). In males, after adjustment for fat mass childhood SEP $\left(0.16 \mathrm{~kg} / \mathrm{m}^{2},-0.07\right.$ to 0.38$)$ and education $(0.09,-0.13$ to 0.30$)$ were not associated with lean mass index, however, low adult SEP was associated with low lean mass index $(-0.21$, -0.44 to 0.01 ). Low SEP was consistently associated with low lean mass index in females after adjustment for fat mass: childhood SEP $(-0.24,-0.42$ to -0.07$)$, education $(-0.36,-0.55$ to -0.17$)$ and adult $\operatorname{SEP}(-0.20,-0.38,-0.03)$.

Conclusion Low SEP across life was associated with high fat mass in early old age. In contrast, low SEP in adulthood in males and across life in females was associated with low lean mass (after adjustment for fat mass). Although further research is required to better understand the pathways underlying these associations, the socioeconomic patterning of these measures is likely to have important public health implications.

\section{OP03 SPATIAL SEGREGATION AND SOCIOECONOMIC INEQUALITIES IN HEALTH IN BRAZILIAN CITIES: COMBINING SPATIAL AND SOCIAL EPIDEMIOLOGY}

doi:10.1136/jech-2012-201753.003

T Chandola. CCSR, University of Manchester, Manchester, UK

Background Spatial and socioeconomic inequalities in health are well-documented across the world, with poorer people, areas and regions experiencing poorer health and higher mortality risks than richer people, areas and regions. Furthermore, greater income inequality has also been linked to higher mortality rates. However, there has been less work on the spatial dimension of such socioeconomic inequalities in relation to inequalities in health. The UNHabitat 2010-11 report on the state of the world's cities identified 
the spatial isolation of poor people in cities (the "spatial poverty trap") as one of the major challenges in developing countries. As people and cities in the developing world get richer, the worry is that the spatial socioeconomic segregation of poor people increases, which in turn may increase their risks of mortality and poor health. Methods Data from 15 major Brazilian cities were analysed, with spatial measures of socioeconomic segregation ("isolation of the poor") estimated for Brazilian districts within cities. The association of the spatial isolation of the poor with district level mortality rates was examined using multiple membership multilevel Poisson regression models to take account of the multilevel (districts within cities) and spatial nature of the data.

Results Increasing spatial isolation of the poor tends to be associated with higher mortality rates, with an interaction between income and spatial isolation. There is not much difference in mortality rates among the poorest districts in terms of spatial isolation. However in the richest districts, districts where the poor are spatially isolated have the highest mortality rates, whereas districts where the poor are not isolated have the lowest mortality rates.

Conclusion As cities in the developing world get richer, there is a risk that this leads to increasing spatial socioeconomic segregation of the poor within those cities. The results from this study suggests that the spatial dimension of poverty within cities may be just as important to health as poverty levels.

\section{OP04 THE ENGLISH NORTH-SOUTH DIVIDE: RISK FACTORS FOR CARDIOVASCULAR DISEASE ACCOUNTING FOR CROSS- SECTIONAL SOCIOECONOMIC-POSITION}

doi:10.1136/jech-2012-201753.004

R Kumar, ARH Dalton. Primary Care \& Public Health, Imperial College London, London, UK

Background Geographic inequalities in health are widely discussed, with an English North-South divide a popular notion. Data indicate the North-South divide in all cause mortality has persisted, even widening in recent years. Given the impact of cardiovascular disease (CVD) mortality on health inequalities, we aimed to assess the extent of a salient North-South divide in risk factors for CVD, controlling for markers of socioeconomic-position (SEP).

Methods We conducted a cross-sectional analysis using the 2006 Health Survey for England using respondents aged 16 years and over. We assessed the population means of systolic blood pressure, total cholesterol, body mass index (BMI) and smoking prevalence. We built nested regression models (all linear regression except for logistic for smoking) adding; demographic (age /sex /ethnicity), SEP indicators (individual income, education attainment, housing tenure, car ownership, occupational classification and area level IMD), behavioural risk factors and vascular disease status. We tested variables for multi-collinearity, assumptions of normality for linear outcomes and use valid survey weights. We finally examine interactions between the North-South divide and age and sex on the risk factors.

Results The North of England showed more deprived characteristics across markers of SEP; except for greater home-ownership. Controlling for demographic variables, we found a significant North-South difference (excess in North) in systolic blood pressure (1.94 [se=0.38]), BMI (0.47 [0.11]) and smoking prevalence $(2.93 \%$ [0.50]). The difference in smoking prevalence was entirely abolished by markers of SEP; both systolic blood pressure and BMI differences were attenuated by SEP, behavioural and vascular disease indicators (1.52 [0.38] \& 0.31 [0.11] respectively), but remained significant. The North-South divide in systolic blood pressure was attributed to differences in men (2.18 [0.54]), being non-significant in women; and in middle age groups (2.70 [0.76] aged 40-59, compared with 2.29 [0.78] aged 16-39) and was non-significant aged 60 and over.
Conclusion Smoking is a major factor behind morbidity and mortality. In line with work from different settings, patterns in smoking can be explained through adverse, cross-sectional patterns of SEP. Addressing underlying poverty and disadvantage may be required to fully tackle smoking inequalities. Using a suite of measures designed to address different constructs of SEP, although cross-sectional, we find excesses in blood pressure and BMI in the North of England. These differences may, in part, explain previously found differences in mortality. If we are to understand, and therefore reduce, geographic inequalities, current measures of SEP may require improvement, for example accounting for aspects of the life-course.

\section{Public Health Interventions: Area and Weight Management}

\section{OP05 FROM TRIAL TO POPULATION: EFFECT OF A WEIGHT MANAGEMENT INTERVENTION ON BODY MASS INDEX WHEN SCALED UP}

doi:10.1136/jech-2012-201753.005

1J Fagg, ${ }^{1 T}$ Cole, ${ }^{2} \mathrm{~S}$ Cummins, ${ }^{1} \mathrm{H}$ Goldstein, ${ }^{3} \mathrm{H}$ Roberts, ${ }^{1} \mathrm{C}$ Law. ${ }^{1} \mathrm{M} R \mathrm{C}$ Centre of Epidemiology for Child Health , UCL Institute of Child Health, London, UK; ${ }^{2}$ Department of Geography, Queen Mary, University of London, London, UK; ${ }^{3}$ General and Adolescent Paediatrics Unit, UCL Institute of Child Health, London, UK

Background The evidence base for effective interventions to manage childhood overweight and obesity is growing. However, results from research may not generalise to service delivery settings, and scaled-up interventions may not reduce health inequalities. We examine a scaled-up childhood weight management intervention, examining variations in body mass index by person, family, place and programme

Methods MEND 7-13 (Mind, Exercise, Nutrition, Do It!) is a multi-component family-based community weight management intervention shown to be effective in a randomised controlled trial (RCT). We used MEND service data from 10,080 children attending MEND programmes from 2007-10. Outcomes were: Change in Body Mass Index (BMI) change over the programme (10 weeks). Anthropometry is measured by trained staff following standardised procedures. We estimated associations between BMI change and participant, family, neighbourhood and programme factors - including pre-specified interactions and random slopes. Multilevel multivariate regression models were used with multiple imputation for missing values. We described participants by BMI at programme start (pre-BMI), age, sex and ethnicity; families by parent-reported employment, lone parent and housing tenure status; places by residential neighbourhood income deprivation, urbanicity, food and built environments; and programmes by the percentage of sessions attended and variables describing programme composition (e.g. group size)

Results BMI was reduced by similar amounts in the RCT and service delivery (RCT BMI change $=-0.91$ [95\%CI: -1.13 to $-0.68]$ ), service BMI change $=-0.75[-0.78$ to -0.73$]$. Service BMI reductions were clinically significant in all socio-demographic groups analysed. However, in multilevel models, pre-BMI, age, ethnicity, unemployment status and programme attendance were independently associated with BMI change. For example, in comparison to white children, reductions in BMI were statistically significantly smaller for black and minority ethnic group children (Asian b=0.29, $\mathrm{p}<0.001$, Black $b=0.20, p<0.001$, (positive coefficients indicate smaller BMI reduction)). Similarly, BMI fell less in children whose parents were unemployed $(b=0.14, p<0.001$, baseline employed parent). There were no significant differences by sex or neighbourhood factors. Tests of interactions and random slopes were non-significant. 